\title{
La cuestión nuclear iraní revisitada: tensiones, sanciones y negociaciones en torno al Plan de Acción Integral Conjunto de $2015^{1}$
}

\author{
The Iranian nuclear issue \\ revisited: Tensions, sanctions \\ and negotiations around the JCPOA
}

\author{
MOISÉS GARDUÑO GARCÍA \\ Universidad Nacional Autónoma de México, México
}

Resumen: El argumento central defiende que la denominada cuestión nuclear iraní debe estudiarse como un fenómeno geopolítico. Aunque el acuerdo nuclear entre el G5+1 e Irán fue bien recibido por la opinión pública internacional como instrumento para "ganarganar", la llegada de la administración Trump mostró intenciones de regresar a un punto donde el conflicto y la tensión imperaran sobre la diplomacia, y esto debido a la amenaza que representa Irán para su política exterior y la de sus aliados en Medio Oriente. Lo anterior implica no sólo el abandono de dicho mecanismo por Washington, sino también el inicio de la estrategia de "máxima presión" para debi-

Recepción: 27 de noviembre de 2017. / Aceptación: 11 de julio de 2018.

${ }^{1}$ Este artículo se realizó con respaldo del Programa de Apoyo a Proyectos de Investigación e Innovación Tecnológica (PAPIT) a través del proyecto IN305119, Sectarismo y justicia social en el Medio Oriente del siglo xxI. 
litar la posición que Teherán ha ido ganando en escenarios precisos de la región, como Siria, Yemen, Iraq y Líbano, estrategia que ha ido acompañada de una narrativa islamofóbica e iranofóbica en la esfera pública global. Desde una perspectiva histórica, se examinan algunos costos de las sanciones para Irán, la inviabilidad de una intervención militar a lo largo de la primera década del siglo y el acercamiento de Israel y Arabia Saudí, que cobró fuerza tras el ascenso de la administración Trump, todo con el propósito de reflexionar sobre los nuevos retos que enfrenta este mecanismo multilateral que ha pasado por ciclos de espirales que incluyen coyunturas de tensiones, sanciones y negociaciones.

Palabras clave: pacto nuclear; tensiones; sanciones; negociaciones; geopolítica.

Abstract: The central argument of this paper defends the view that the Iranian nuclear issue must be studied as a geopolitical phenomenon. Although the nuclear deal was welcomed by the international community, the arrival of the Trump administration caused a return to a dynamic of conflict and mistrust. Washington's withdrawal from the nuclear deal marks the beginning of the "maximum pressure strategy" to weaken Tehran in specific theaters such as Syria, Yemen, Iraq, and Lebanon, which includes a narrative of Islamophobia and Iranophobia in the transnational public sphere. From a historical perspective, this text examines some costs of sanctions for Iran, the unfeasibility of a military intervention during the first decade of the 21st century, and the strategic approach of Israel and Saudi Arabia during the Trump presidency, all in order to offer insights into the challenges facing the nuclear deal through spiraling cycles of tensions, sanctions, and negotiations.

Keywords: nuclear deal; tensions; sanctions; negotiations; geopolitics.

\section{El programa nuclear iraní como cuestión geopolítica}

Desde 2003, la controvertida cuestión nuclear comenzó a pensarse como detonante potencial de una nueva guerra en el 
golfo Pérsico. El problema radicó en el disenso de percepciones que dicha reactivación generó entre las partes, pues, en primer lugar, la República Islámica defendió que su programa nuclear tenía fines pacíficos, mientras Israel, Arabia Saudí y Estados Unidos, así como grupos de derecha en Francia y otros países europeos, declararon que Teherán tenía "claros propósitos militares" (Parsi, 2007). A pesar de que ningún servicio de inteligencia ni los inspectores del Organismo Internacional de Energía Atómica (OIEA) pudo demostrar que Irán orientara sus actividades nucleares hacia fines bélicos, se impuso un fuerte régimen de sanciones económicas al gobierno de Teherán para obligarlo a abandonar sus pretensiones nucleares. Esta situación fue vista por Irán como un ejemplo de discriminación por parte de Washington, al citar las diferencias con que trataban a otras naciones con capacidad nuclear que no eran firmantes del Tratado de No Proliferación Nuclear (TNP) y a las cuales, sin embargo, no se les exigía el grado de inspección que se demandaba a la República Islámica. Tales eran los casos de India y Pakistán, países que contaban con cientos de ojivas nucleares en sus respectivos arsenales, que no eran parte del régimen internacional de no proliferación nuclear en el mundo y que, no obstante, gozaban de plenas relaciones de cooperación estratégica con Estados Unidos, lo que sugería altos grados de tolerancia a la posesión de armas de destrucción masiva (U.S. Department of State, 2007 y 2015a).

Vale la pena reflexionar, entonces: si Irán ya era uno de los 191 países firmantes del TNP y uno de los 93 que lo habían ratificado (United Nations Office for Dissarmament Affairs, 1970), "¿por qué se bloqueaba su derecho a enriquecer uranio mientras este mismo derecho se le garantizaba a países como India, Pakistán o Israel, los cuales (aparte de no haber firmado el TNP) contaban con una fuerte influencia de los altos mandos militares en sus respectivos programas nucleares?" (Barzegar, 2008a).

En efecto, la cuestión nuclear iraní no se trataba de un asunto relacionado con la falta de informes al OIEA, sino de uno 
estrictamente geopolítico que ponía en tela de juicio el modelo de dominación e influencia económica y política proyectado por Estados Unidos e Israel en el Medio Oriente en general, y por Arabia Saudí en el golfo Pérsico en particular. Y es que Irán, debido al alza de precios del petróleo y el gas que se experimentó de 2005 a 2008, no sólo logró posicionarse como un Estado rentista que consolidó una fuerte base social gracias a los subsidios a la gasolina y otros productos de consumo básico entre la población, sino que además se convirtió en una de las naciones de Medio Oriente fabricantes (y posteriormente exportadoras) de cohetería y misiles de largo y mediano alcance. Un ejemplo de esta evolución tecnológica fue la puesta en órbita de su primer satélite (Omid, “esperanza” en lengua farsi) en 2009 (Alizadeh, 2014, pp. 76-111).

El beneficio del rápido crecimiento de los ingresos del petróleo le permitió a Irán incrementar su ayuda a movimientos de base islámica, como el Yeish al-Mahdi en Iraq, Hamas en Palestina y Hezbollah en Líbano (considerados como organizaciones terroristas por Washington), además de desempeñar un papel influyente en la reconstrucción de Iraq tras el desarrollo de infraestructura multimodal, petrolera, energética y comercial a través de un corredor económico que se inicia en la zona de Basra y se conecta mediante una red de carreteras, hoteles, gasolineras y tiendas de productos básicos con la parte central de Bagdad, ciudad a la que le surte $80 \%$ de su energía eléctrica desde 2007 y con lo cual genera, junto con el resto de las actividades comerciales bilaterales, un aproximado de 5000 millones de dólares anuales en promedio (Barzegar, 2008b, pp. 47-48; Rahimi, 2012, p. 25).

Así, en un contexto caracterizado por la intervención estadounidense en Iraq y en Afganistán, la difusión de la metanarrativa de la cruzada contra el terrorismo impulsada por la doctrina Bush, la creación de un grupo de países denominado "Eje del Mal" (del que Irán era parte), las ofensivas retóricas de Israel, que acusaba a Irán de ser una amenaza para su seguridad nacional, además del insistente discurso conservador en Estados 
Unidos a propósito de un cambio de régimen en Teherán, era claro que las actividades económicas, políticas y estratégicas de Irán en el mundo árabe representaban un desafío al modelo del Gran Medio Oriente proyectado por George W. Bush en la Cumbre del G8 de Sea Island, Georgia, desde 2004.

Por la parte iraní, la invasión a Iraq en 2003 hizo pensar a diversos cuadros de los Pasdaran que su país podría estar en la lista de naciones susceptibles de ser intervenidas en el corto plazo, lista a la que se sumarían Líbano y Palestina en 2006 y 2008, respectivamente, y otros, como Siria, Libia y Yemen, que serían intervenidos tras las revoluciones árabes, hechos que se confirmarían con el paso del tiempo y que respaldarían las preocupaciones de las élites iraníes más conservadoras. ${ }^{2}$

Con esto, son dos los puntos importantes para hablar del programa nuclear de Irán como un asunto geopolítico: primero, las implicaciones meramente económicas del mix energético, y segundo, aquel que se basa en las repercusiones del elemento disuasivo. Atendiendo al primer factor, un argumento para el desarrollo de energía nuclear en los países exportadores de petróleo es que, "mediante la incorporación de la energía nuclear al mix energético, toda la producción de petróleo y gas de la industria nacional puede dirigirse al sector externo (y usarse la energía nuclear sólo para consumo interno), lo que genera un mayor beneficio económico a mediano y largo plazos" (Wood, Milazzo, Reichmuth y Bedell, 2007, p. 89). Rusia,

${ }^{2}$ La historia reciente de las relaciones entre Irán y Estados Unidos muestra evidencias que fortalecen las preocupaciones de Teherán, entre ellas: el golpe de Estado de la CIA (Agencia Central de Inteligencia) contra Mohammed Mossadeq en 1953; el intento de sabotear la Revolución islámica de 1979; el apoyo a Francia para no devolver dinero iraní después de dicha revolución; el apoyo incondicional a Saddam Hussein durante la guerra Irán-Iraq a lo largo de la década de 1980; el derribo "por error" del avión civil de la aerolínea Iran Air (vuelo 655) por parte del USS Vincennes cuando patrullaba el golfo Pérsico el 3 de julio de 1988; el apoyo a las actividades de espionaje a través de grupos considerados como organizaciones terroristas por Irán, como el Moyahedin-e Jalq-e Iran; la traición de la administración Bush cuando Irán ayudó a sofocar al régimen talibán en Afganistán y Washington no le reconoció el papel de mediador y pacificador en la región y en su lugar lo agregó a la lista de países miembros del Eje del Mal, entre otros (Abrahamian, 2015). 
por ejemplo, ha avanzado en esta lógica y ha logrado producir grandes excedentes económicos de la venta de gas natural para financiar la producción y la exportación de armamento a gran escala, estrategia que siguió inmediatamente después de sacar a la élite atlantista del poder ante la llegada de Vladimir Putin en el año 2000.

De acuerdo con expertos en cuestiones técnicas, este argumento es válido en la medida en que el petróleo, el gas y la energía nuclear son tecnológicamente sustituibles entre sí, sobre todo para la generación de electricidad, siempre y cuando el costo del programa nuclear no esté por encima de las expectativas de exportaciones petroleras o de gas natural que se consideren a mediano plazo a partir de cálculos del comportamiento de los precios en el mercado. En el caso de Irán, hay que decir que su programa nuclear no ha sido nada barato, pues tan sólo el gasto de los contratos entre Irán y las diversas empresas rusas (Atomstroyexport, Minatom, Atommash y Rosatom) que han ayudado a remodelar el reactor de Bushehr desde los años noventa y hasta su inauguración en 2010 fue de alrededor de los 11000 millones de dólares, según algunas estimaciones (Wood et al., 2007, p. 90; Vaez y Sadjapur, 2013, p. 5). ${ }^{3}$ Esta inversión se entiende si se piensa que Irán no sólo tiene reservas petroleras probadas por 90 años al ritmo de producción de 2018, sino también reservas de gas natural probadas por un estimado de 220 años, lo que lo convierte en uno de los países con más reservas de hidrocarburos en el mundo y con una expectativa de renta alta y sostenida. Con estos datos es posible argüir que Irán también podría haber buscado el control del conocimiento del ciclo del combustible nuclear (know bow of fuel cycle) con el objetivo a largo plazo de que el sector se dedicara a abastecer el mercado energético interno para sostener un nivel alto de exportación de hidrocarburos, sobre todo enfocado en la demanda del mercado asiático de países como China, Japón o India, que, aunque con programas de energía nuclear, tienen

${ }^{3}$ Los primeros involucrados en este proyecto fueron Alemania y Francia. 
una alta demanda petrolera para satisfacer la industria y el sector tecnológico que sustentan su crecimiento económico.

El segundo eje de discusión para estudiar el programa nuclear iraní como problema geopolítico radica también en el ciclo del combustible nuclear, pero visto como elemento $d i$ suasivo. El argumento es el siguiente: el control del ciclo del combustible es, incluso, un elemento mucho más valioso para Teherán que la misma bomba, porque cuando se obtiene la fórmula completa para enriquecer uranio (sin importar a qué porcentaje se haga), se accede a la capacidad de usarlo de la misma forma en que se usaría un artefacto nuclear (aunque sin contar con él). Esto se conoce como opacidad militar, la cual disuade a los enemigos externos (por el potencial que se tiene de hacer un arma en cualquier momento), al tiempo que se respetan los márgenes de legalidad impuestos por los regímenes internacionales (porque, materialmente, no se cuenta con un artefacto nuclear) (Mesa, 2009, p. 834).

Mientras el camino al mix energético iraní se respalda con gran cantidad de reservas probadas de gas y petróleo, el que lleva a la capacidad disuasiva se apoya en el mencionado programa de misiles, ambas esferas controladas por los Pasdaran, el órgano militar más confiable y mejor preparado de la República Islámica de Irán, lo cual, a su vez, también levanta sospechas entre los críticos estadounidenses, israelíes y saudíes acerca de los propósitos del programa nuclear iraní (Forozan, 2016, p. 250).

Los elementos anteriores conforman y confirman una estructura geopolítica que puede verse como un juego de suma cero en el que, para los actores en disputa, lo que está de por medio es el control y la institucionalización de áreas de influencia económica y de distribución de recursos no sólo del golfo Pérsico, sino también del mundo árabe (Barzegar, 2010, p. 75). Esta estructura geopolítica explica, al menos en cierto grado, las alianzas entre Rusia y China, Irán, Siria y Hezbollah por un lado, y de Estados Unidos con Arabia Saudí e Israel, por el otro, coaliciones dinámicas que se ajustan periódicamente y 
que no son estáticas, pero que ante la estructura de los intereses señalados se mantienen estables, y las evidencias para sostener este argumento son los enfrentamientos en campos de bata1la, como la cuestión palestina, la guerra en Líbano de 2006, la reconstrucción de Iraq y, muy recientemente, las crisis humanitarias en Siria y Yemen.

\section{Las tensiones alrededor de la cuestión nuclear: entre la opción militar y el régimen de sanciones}

La cuestión nuclear en esa estructura geopolítica estuvo rodeada de una retórica desafiante de las partes involucradas, y el intento de normalizar la satanización del programa nuclear en los medios globales de comunicación, al menos en los occidentales, es una muestra. Sobre todo al recurrir a la repetición de un vocabulario específico, con expresiones como iranofobia, islamofobia, armas de destrucción masiva, bombing Iran, entre múltiples formas gráficas que parecían preparar a buena parte de la opinión pública para una nueva guerra en la región, tal como se había logrado con Iraq y Afganistán tras los acontecimientos del 9/11 (Torabian y Abalakina, 2012, pp. 463-478). Citaré dos ejemplos: George W. Bush aceptó en sus memorias haber pensado en un ataque militar directo contra Teherán junto con operaciones paralelas en Siria durante su segundo mandato (Bush, 2010, p. 497). Netanyahu declaró en varias ocasiones: "Haría todo lo que estuviera en mis manos, incluso ir a la guerra solo contra Irán, con tal de parar las pretensiones nucleares de los islamistas radicales contra mi país" (Benari, 2012). Por su parte, Mahmoud Ahmadineyad, en un discurso ante la Asamblea General de la ONU, en septiembre de 2012, habló de "la necesidad de erradicar el sionismo de la región", citando palabras de Jomeini, las cuales fueron mal interpretadas por la prensa occidental al traducir su discurso con frases como "es necesario borrar a Israel del mapa del Medio Oriente”, lo que no ayudó en nada a apaciguar el ambiente tenso, ni siquiera en 
foros diplomáticos como el que ofrecía Naciones Unidas y algunos de sus organismos (Charbonneau, 2012).

Al momento de estas declaraciones, algunos expertos en la región estuvieron de acuerdo en los efectos catastróficos que tendría una hipotética intervención en Irán y en la inviabilidad estratégica que implicaba. Como evidencias para este argumento están los análisis de Colin Kahl (2012) en Foreign Affairs (en respuesta a intelectuales de derecha en Estados Unidos, como Matthew Kroenig [2012], que abogaban por el ataque inmediato), cuando abordó la forma en que dicho fenómeno habría retrasado la salida de tropas estadounidenses de Iraq, y cómo habría preparado un terreno fértil para la insurrección de protestas populares en Estados Unidos, tal como había ocurrido con Vietnam años atrás. En trabajos previos se ha estudiado, desde el punto de vista militar, la poca efectividad que una intervención quirúrgica militar tendría sobre Irán (Garduño, 2011, pp. 72-73). Por ejemplo, es necesario recordar que los blancos potenciales de ataques aéreos se encuentran dispersos a lo largo y ancho del país, por lo que serían necesarios varios ataques contra instalaciones estratégicas en Isfahán, Bushehr, Teherán y Arak, donde los aviones extranjeros y los buques portamisiles se enfrentarían a las bases más efectivas de misiles tierra-aire con los que cuenta Irán, al haber sido diseñados con ayuda rusa para ese tipo de contingencias. Otro elemento por rescatar es la estratégica ubicación del reactor nuclear de Bushehr, colocado en las costas del golfo Pérsico no sólo para garantizar el suministro de agua de mar para refrigerar las vainas de combustible en caso de emergencia o accidente, sino también como factor para disuadir ataques en su contra, pues de llevarse a cabo causarían un accidente catastrófico en las aguas del golfo que afectaría el hábitat de la fauna marina y los intereses económicos de las industrias petrolera y gasera, así como de la flota mercante de los países que cuentan con litoral o negocios en la zona, particularmente las petrocracias árabes.

Un ejemplo más de la poca efectividad de una intervención militar en Irán es la respuesta que Teherán ha insinuado en el 
estrecho de Ormuz, que si bien no lo cerraría por completo, ciertamente estaría determinado por una lucha asimétrica ensayada por la Marina del ejército regular iraní (Artesh), y en particular por el ejército irregular (Pasdaran), los cuales llevan años adiestrándose conjuntamente en cinco grandes zonas marítimas del país, tres de ellas en el golfo, es decir, Bandar Abbas, Bushehr y Kish, para hacer frente a los ejercicios militares que, por su parte, la Quinta Flota Naval estadounidense ha realizado durante años con base en Bahréin (Una guerra entre Irán y Estados Unidos en el Golfo Pérsico, 1987; Cordesman, 2007, p. 2; Garduño, 2012, p. 8). ${ }^{4}$

La retórica de confrontación recibió duras críticas ante los límites de la opción militar. En junio de 2009, Benjamín Netanyahu fue criticado por Aharon Zeevi Farkash, exministro de Inteligencia Militar de Israel, quien describió la percepción pública israelí como “distorsionada” (Bergman, 2009). El final del mandato de George W. Bush estuvo marcado por una crítica similar, al grado de recibir ataques en público, como el del periodista iraquí Muntazar al Zaidi, quien le lanzó un par de zapatos al mandatario estadounidense en 2008 durante una conferencia de prensa en Bagdad, acción que Mahmoud Ahmadineyad también experimentaría durante su visita a El Cairo en 2013 (Chumley, 2013).

${ }^{4}$ Los ataques de misiles estadounidenses contra Siria en abril de 2017 y 2018, respectivamente, mostraron un avance en la capacidad disuasiva de Washington. Esto se apreció cuando, el 14 de abril de 2018, el general James Mattis anunció que el "golpe de castigo" contra el presunto ataque con armas químicas en la zona de Duma, Siria, sería de "un solo tiro" y alcanzó tres puntos de diversas zonas geográficas del país árabe con ayuda de Francia, Reino Unido y Qatar (país que prestó su espacio aéreo para lanzar misiles desde el golfo Pérsico). Si bien el mensaje de Washington se leyó como la misión de golpear tres sitios simultáneamente con un solo disparo, en el mismo escenario sirio el ejército regular iraní y las milicias afganas que comandan las fuerzas revolucionarias de los Pasdaran también han ganado experiencia en batalla al enfrentarse a milicias islamistas respaldadas por Arabia Saudí y otros poderes fácticos, como el Estado Islámico y grupos pertenecientes a $\mathrm{Al}$ Qaeda. Lo anterior hace que la paridad estratégica se mantenga en términos de guerra asimétrica, estrategia a la que siempre le ha apostado Teherán dado el ambiente tenso que vive la región, al menos desde la guerra Irán-Iraq de los años ochenta. 
La retórica injerencista no cesó durante la llegada de Obama y Rohaní a la presidencia de sus respectivos países, y estuvo acompañada del fortalecimiento del régimen de sanciones que se había impuesto a Irán desde décadas anteriores. Al respecto hay que decir que Irán ha recibido tres tipos de sanciones: las impuestas por Estados Unidos, las provenientes de la Unión Europea y las consensuadas en el seno del Consejo de Seguridad de Naciones Unidas (CSNU) (Samore, 2015, pp. 1-18). De éstas, las aplicadas a partir de 2008 estuvieron dirigidas a los sectores energético y financiero para expandir el embargo petrolero estadounidense de 1995 hacia otros países que compraban petróleo iraní, como los de la Unión Europea, India y China, y para evitar la inversión extranjera en negocios concernientes al gas, el uranio y el transporte multimodal. Por su parte, las aplicadas con rigor a partir de 2011 fueron las que más lastimaron las finanzas iraníes al aislar su red de bancos del sistema financiero internacional, incluido el Banco Central de Irán, lo que obstaculizó sus transacciones, bloqueó el sistema SWIFT (Society for Worldwide Interbank Financial Telecommunication), monitoreó transacciones tipo U-Turn, congeló cuentas bancarias en el extranjero que sumaban cerca de 50000 millones de dólares y designó las actividades de prominentes líderes de los Pasdaran como "peligrosas para la economía mundial" (Samore, 2015, p. 17).

Aunque estas sanciones estaban dirigidas contra la élite más influyente de la economía del país, es sabido que las consecuencias de este tipo de mecanismos suelen ser más severas para la población del país sancionado, al elevarse los precios de los bienes relacionados con el petróleo y de aquellos provenientes del extranjero, o como ocurrió en el caso de Irán con el aumento del precio de algunas medicinas para enfermedades como el cáncer o la esclerosis, medicamentos que provienen en su mayoría de farmacéuticas europeas (Dehghan, 2012). Así, ante la referencia de las sanciones a países como Iraq o Cuba, el caso de la República Islámica podría estudiarse como un mecanismo que tenía la intención de polarizar a la población para aprove- 
char la crisis nacional de 2009 después de la polémica reelección de Ahmadineyad, e impulsar una nueva salida de los jóvenes a las calles para poner en aprietos al gobierno. Sin embargo, la estrategia en esa dirección nunca funcionó, porque en lugar de crear insurrecciones internas, las sanciones fueron manejadas por el gobierno iraní como "un mal proveniente del exterior" que había que atacar en conjunto mediante una economía de resistencia, al grado de reapropiarse de la estrategia y usarla, al igual que la retórica de la confrontación, como instrumento de cohesión interna (Khamenei, 2014).

No obstante que algunas sanciones tuvieron efectos profundos, no todas funcionaron. En el rubro energético, por ejemplo, aunque afectaron las exportaciones petroleras, el crudo iraní siguió beneficiándose de los altos precios del mercado internacional (hasta que bajaron a finales de 2014). Algunas empresas, como Total (Francia) y ENI (Italia) se retiraron del territorio iraní y dejaron de venderle gasolina a Teherán, pero el hueco que dejaron fue llenado poco después por compañías como Petróleos de Venezuela, Royal Oyster Group (de Emiratos Árabes Unidos), Tanker Pacific (Singapur), Allvale Maritime (subsidiaria de Ofer Brothers Group, de Israel) y Associated Shipbroking (Mónaco), entre algunos otros particulares del Kurdistán iraquí que se dedicaban al mismo rubro y que abastecieron a Irán de los refinados que necesitaba, con lo que facilitaron el proceso que lo llevó a declararse productor y exportador de gasolina en septiembre de 2010, cuando comenzó a venderla al gobierno central de Iraq y a otros países vecinos, como Armenia y Afganistán, por medio de la National Iranian Oil Company (Garduño, 2011; Katzman, 2011, pp. 9-11; Reuters, 2010).

La medida que más dañó a Irán fue la marginación del Banco Central de Irán del sistema financiero internacional, pues se trata de la institución de donde se obtienen los pagos derivados de las exportaciones del petróleo (Habibi, 2013, p. 3). Este bloqueo obligó a Teherán a sustituir el dólar por las monedas nacionales de algunos clientes, como Turquía, India y China, para luego convertirlas en oro y así transferirlo a 
Turquía, y de ahí a Dubái (registrándolo como oro turco), y ser posteriormente reexportado a Irán o usado para comprar bienes de importación (Motamen-Samadian, 2014, pp. 136-138). Esta triangulación condujo a un aumento sospechoso en las exportaciones de oro de Turquía en la primera mitad de 2012, lo que atrajo la atención de Estados Unidos (pues Turquía no figura como un fuerte productor internacional de oro). A finales de ese año, Estados Unidos prohibió las exportaciones de oro a Irán, lo que, junto con la disminución de los precios del petróleo en 2014, resultó en la devaluación del rial iraní hasta en $50 \%$, que a su vez provocó fuga de capitales a Europa y a Asia (Salehi-Isfahani, 2013).

A pesar de la devaluación, Irán tuvo productos que se vendieron a menor precio pero en mayor cantidad en mercados no occidentales, como ocurrió con las alfombras persas, un sector que emplea a aproximadamente seis millones de personas en todo Irán y que aporta cerca de 3.5\% del PIB nacional. El dato se toma de la información proporcionada por la Industria $\mathrm{Na}$ cional de Alfombras de Irán en 2011: "A pesar de perder mercado en Estados Unidos, hubo un aumento en las exportaciones de hasta $49 \%$ en otros países, como China" (sobre todo en la clase media emergente), lo que representaba un valor estimado en 500 millones de dólares (Handmade carpet exporters look to China market, 2011).

Si bien Irán no es miembro de la Organización Mundial de Comercio, ha apostado por la diversificación comercial, y en un mundo donde hay más procesos de regionalismo que Estadosnación (cerca de 400 mecanismos regionales contra 193 Estados en el sistema internacional), y donde $60 \%$ del comercio mundial tiene lugar entre bloques comerciales regionales, amplió sus contactos con bloques como la Organización de Cooperación Económica (ECO, por sus siglas en inglés), el Sistema Global de Preferencias Comerciales entre Países en Desarrollo, el G-8 de los países islámicos en vías de desarrollo, el G-15, la Asociación Rim del Océano Índico para la Cooperación y la Asociación Regional y la Organización de la Conferencia 
Islámica, entre otros, donde es miembro observador, como en la Organización de Cooperación de Shanghái (UNCTAD, 2013). Además, Irán ha contado con más de 60 instrumentos de inversión y la red más grande de zonas económicas especiales y de libre comercio de todo el golfo Pérsico para paliar las sanciones comerciales y el bloqueo a sus productos (Hakimian, 2014)..$^{5}$ No obstante, una debilidad de esta red de acuerdos radicó en el denominado e-business, pues los turistas que visitan Irán, por ejemplo, no pueden usar las tarjetas de crédito y débito Visa y Mastercard para realizar compras. Las mayores barreras a este tipo de comercio en Irán han sido: infraestructura (terminales bancarias, plásticos), desconfianza hacia el comercio electrónico, falta de capacitación del personal en cuanto a leyes en ese campo e instalaciones relacionadas con internet de alta velocidad.

Finalmente, cabe destacar que las sanciones a la aviación civil y a la industria automotriz fueron sumamente efectivas. Ambos sectores fueron los más frágiles desde los inicios de la revolución. Por ejemplo, desde 1979 fue casi imposible modernizar la principal aerolínea, Iran Air, sobre todo en cuanto a refacciones y mantenimiento. En 2010, ante la negativa de empresas extranjeras de proveer combustible para viajes de retorno en aeropuertos europeos, Iran Air entró en una de sus crisis más recientes que estuvo a punto de llevarla a la quiebra (Iran to sue European refusal to refuel planes: Airline, 2010). Lo anterior se tradujo en, al menos, un accidente aéreo al año, aunque en 2014 se reportaron otros en marzo, mayo y agosto, respectivamente (Iranian airplane crashes expose weaknesses in 'selfsufficiency', 2014).

En el sector automotriz, en 2012 hubo una baja respecto al año anterior de casi $32 \%$ en la producción de unidades en empresas como Peugeot, Kia, Hyundai, Daewoo, Nissan y Renault, por lo que un serio desabasto de refacciones causó grandes

\footnotetext{
${ }^{5}$ Respecto de las zonas económicas especiales de Irán, véase https://www. freezones.ir/
} 
pérdidas en una de las áreas más importantes de la economía iraní, el bazar, que tuvo que echar mano de otras fuentes de distribución cercanas a las rutas de contrabando y los mercados clandestinos, por lo que algunas prácticas se volvieron ambivalentes entre los comerciantes establecidos (bazaaris) y los no establecidos, al transgredir las categorías de "comercio lícito" e "ilícito", en una especie de comercio híbrido que no era ilegal, sino tolerado para hacer frente a las sanciones externas, lo que ayudó en gran medida a mantener una dinámica en el mercado de consumo interno (Erami y Keshavarzian, 2015, pp. 110-139).

Cerca del Plan de Acción Integral Conjunto (PAIC) de 2015: el factor coyuntural y la flexibilización de algunos temas clave para la negociación

La mayoría de los análisis del Plan de Acción Integral Conjunto (Joint Comprehensive Plan of Action) coinciden en que el pacto con Irán respondió tanto a las posibilidades nulas de intervenir militarmente como al efecto limitado de las sanciones. Sin embargo, esta explicación queda incompleta si no se conecta con elementos coyunturales como el estallido de las revoluciones árabes en 2011 y los efectos de la crisis de 2008 en Medio Oriente, fenómenos que sacudieron no sólo la cuestión nuclear, sino también otras dinámicas de conflicto ligadas a la estructura geopolítica descrita.

Hamid Dabashi propone pensar la cuestión nuclear considerando la combinación de los factores geopolítico-estructurales con aquellos de índole coyuntural, donde se inserta el estallido de las revoluciones árabes, y la forma en que dicha combinación afectó a las sociedades y los regímenes de las partes en disputa. Dabashi se refiere a este ambiente como

[...] una atmósfera donde no hay una guerra declarada de jure, pero sí una amenaza externa y potencial que sirve para justificar de facto una 
militarización de la política con la cual se pueden controlar posibles brotes de insurrección interna. A su vez, esta militarización de facto sirve para justificar las acciones en el ámbito externo, esto debido al descontento popular presentado por la precaria situación económica en el mundo y distintos ejercicios de opresión y falta de canales de participación política en el mundo árabe (Dabashi, 2011, p. 170).

Esta propuesta sirve para reflexionar sobre las estrategias que los gobiernos de Estados Unidos, Irán e Israel, por ejemplo, comenzaron a poner en marcha ante las protestas del Occupy Wall Street Movement, las del Yanbesh Sabzi y las del Housing Protest, respectivamente, que habían brotado en plena coincidencia con lo que estaba ocurriendo con las revoluciones en todo el Medio Oriente (Dabashi, 2011, p. 174).

En este estado de guerra no sólo se invisibilizaron las protestas en los grandes medios de comunicación, también se nubló la cobertura de la intervención de Israel en Gaza en 2012, de los efectos de la crisis de 2008 en Washington y en los países europeos del Mediterráneo, además de la represión de Irán al Movimiento Verde en las protestas de febrero de 2011. Esta reflexión se podría extender a los países árabes del golfo Pérsico que querían evitar que la ola de protestas llegara a sus sociedades, al grado de ver con buenos ojos la intervención saudí en Bahréin en marzo de 2011 para evitar colapsos mayores en aquella zona (Joyce, 2012, p. 169).

La estrategia de cohesionar a la población culpando al exterior de los problemas económicos es clásica y permite justificar altos presupuestos militares, posicionar a grupos políticos conservadores en sus respectivos regímenes e inundar los medios con noticias que alimentan la esfera pública y aumentan el rating. El armamento que se produjo en este contexto no se utilizaría para iniciar guerras directas, sino para echar a andar operaciones militares encubiertas que tendrían el objetivo de mantener el statu quo en la región mediante ataques selectivos y calculados en un contexto que tomó a los actores por sorpresa (Sandler y George, 2016, p. 174). Como ejemplos se recuerdan el asesinato de cinco científicos iraníes entre 2010 y 2012 en Te- 
herán, donde el gobierno iraní acusó a Israel de la autoría (Espinosa, 2012); el asesinato de cinco ciudadanos israelíes en la explosión de un autobús en el aeropuerto de Burgas, Bulgaria, en julio de 2012, del que Israel acusó a Hezbollah e Irán (Associated Press, 2012); o los múltiples casos de provocación entre la Marina de Estados Unidos y la Marina iraní entre 2012 y 2016 en las aguas del golfo Pérsico (Alandete, 2012; Irán libera a los infantes de la Marina de EE.UU. detenidos en el Golfo Pérsico, 2016).

La prioridad para algunos mandatarios regionales fue evitar que las protestas se expandieran. Esto provocó una inmediata militarización de los espacios públicos y un lenguaje sectario con muestras explícitas de violencia que inundó los medios de comunicación y sustituyó el principal, hasta ese entonces, tema de la agenda internacional: la cuestión nuclear. La intervención militar de la Organización del Tratado del Atlántico Norte en Libia en 2011; la mencionada intrusión saudí en Bahréin en marzo de 2011 (y luego la de marzo de 2015 en Yemen); el apoyo armado y económico de Irán y Arabia Saudí a grupos como Hezbollah y el entonces llamado Frente al Nusra; la nueva injerencia militar de Israel en Gaza en 2012 (y en 2014), la exacerbación del conflicto entre Turquía y los kurdos del Partido de los Trabajadores del Kurdistán (PKK) y una retórica de "nueva Guerra Fría” entre Rusia y Estados Unidos en la cuestión siria son evidencias de este argumento.

El militarismo regional fue, paradójicamente, el contexto de las nuevas rondas de negociación de la cuestión nuclear. A partir de noviembre de 2013 se alcanzaron los principales acuerdos para "evitar que Irán se hiciera de un artefacto nuclear, con la garantía al mismo tiempo de su derecho a enriquecer uranio" (Borger y Dehghan, 2013). Y si las posibilidades de intervención militar en Irán eran escasas antes de 2011, después de las revueltas populares árabes fueron casi nulas; Teherán había pasado de 3000 centrifugadoras de uranio conectadas en mayo de 2007 a casi 19000 en abril de 2015 (Davenport, 2015). Además, con la defensa de los intereses rusos en Siria, el 
apoyo de Moscú a más sanciones internacionales contra Irán en el seno del CSNU se congeló (Tussaint, 2012). Condiciones de carácter local, regional y global coincidieron entonces para que Irán y los miembros del G5 + 1 lograran un pacto con beneficios financieros para todos, en lugar de promover una nueva guerra en esa región de por sí convulsionada por la dinámica de conflictos que preocupaba a muchos grupos en el poder ( $\mathrm{Ta}$ rock, 2016, p. 1411).

La cuestión nuclear entró en una nueva fase cuando Barack Obama le dio la bienvenida al triunfo electoral de Hassan Rohaní en Irán en junio de 2013. La mediación de Omán y el interés económico de Alemania fueron elementos centrales para organizar nuevas rondas de negociación (Gupta, 2015). Un discurso de paz se hizo necesario ante la insurrección de poderes fácticos como el Estado Islámico y el uso de armas químicas en el terreno sirio. En este contexto se retomó incluso la propuesta de la construcción de una zona libre de armas nucleares en Oriente Medio, producto de experiencias similares en muchas regiones en el mundo, principalmente en la latinoamericana, como una herramienta para allanar el camino a la estabilización de la región y para contribuir a la paz internacional (Taylor, 2013).

Con esto se flexibilizaron algunos temas, como el número de centrifugadoras de primera y última generación que se podía tener habilitadas, el levantamiento de sanciones financieras y el descongelamiento de fondos iraníes en el extranjero, el stock de enriquecimiento de uranio (de bajo enriquecimiento y el enriquecido a 20\%), el destino de las instalaciones de Fordo, la sistematización de las inspecciones para Irán y lo relacionado con el reactor de agua pesada en Arak.

Respecto a las cuestiones técnicas, después de alcanzar un acuerdo preliminar el 24 de noviembre de 2013, en el que se sentaron las bases de temas clave, como la suspensión del enriquecimiento de uranio, la reducción del número de centrifugadoras y el levantamiento gradual de algunas sanciones económicas, comenzó una serie de 13 rondas de negociación que 
sería el camino para llegar a la firma final del PaIC del 14 de julio de 2015. En ese documento, las partes dispusieron que Irán estaría durante casi 25 años bajo inspección exhaustiva del OIEA (ningún miembro del TNP ha estado en dicho régimen de revisión nunca en la historia), ${ }^{6}$ que reduciría el uso de dos terceras partes de sus centrifugadoras de primera generación (de 18472 a solo 6104, incluidas las 1044 que se instalarían en el futuro Centro de Tecnología e Investigación de Fordo), que desconectaría todas las centrifugadoras de última generación, que no haría investigación científico-tecnológica en nuevas generaciones de centrifugadoras, que la cantidad de uranio de bajo enriquecimiento que mantendría al menos durante 15 años no sería mayor a los $300 \mathrm{~kg}$ y, además, que podría enriquecer uranio a no más de un promedio de entre 3 y $7 \%$ anual por un mínimo de 10 años (U.S. Departament of State, 2015b, pp. 1-18).

Lo que se solicitó en la discusión de estos temas fue la garantía de que Irán no tendría la capacidad (y no sólo la intención) de crear un arma nuclear, mientras que Teherán esperaba ver reconocido su derecho a enriquecer energía nuclear y el levantamiento de las sanciones financieras para tener acceso a fondos de casi 100000 millones de dólares congelados hasta ese momento en bancos occidentales.

$\mathrm{Al}$ respecto, hay que hacer algunas consideraciones interesantes, pues el análisis del pacto no se reduce a la explicación mencionada. En primer lugar, hay que pensar en las agendas internas y en la forma en que el pacto fue presentado a una opinión pública global que tanto había estado expuesta a una re-

${ }^{6}$ El OIEA tiene la responsabilidad de supervisar y verificar las disposiciones en materia nuclear del acuerdo avisando a Irán al menos con 24 días de antelación, previo acuerdo de los miembros del CSNU y Alemania. El pacto proporciona actualizaciones periódicas a la Junta de Gobernadores del OIEA y al CSNU. El OIEA tiene un equipo de no más de 150 inspectores designados para Irán. Según el acuerdo, Irán "generalmente debe permitir la designación de los inspectores cuando sean provenientes de los países que tienen relaciones diplomáticas con Teherán" (es decir que Irán prohibiría a inspectores de Estados Unidos e Israel además de ejercer poder de veto sobre ciertos inspectores que considere no adecuados); véase Chin y Lincy, 2015. 
tórica de guerra y confrontación. Irán pudo vender el pacto a la sociedad iraní como "un triunfo contra la arrogancia de Occidente" al lograr el reconocimiento como potencia nuclear en el Medio Oriente y como un país con derecho a enriquecer uranio como miembro firmante del TNP (Rouhani: Iran has achieved all its 4 objectives in nuclear talks, 2015). Por su parte, la administración de Obama presentó el pacto como "el camino perfecto para evitar una guerra más en el Medio Oriente y, al mismo tiempo, obstaculizar que Irán se hiciera de una bomba nuclear" (Full text Obama gives a speech about the nuclear deal, 2015).

Con la firma del PAIC, el Banco Mundial publicó un informe sobre las implicaciones económicas del levantamiento de las sanciones a Irán. Con base en la reactivación de la venta de crudo a la Unión Europea, la reducción en los costos de transporte en el comercio con Irán y el incremento de la productividad del comercio transfronterizo de servicios, asentó, Teherán produciría unos 13000 millones de dólares anuales tan sólo durante la primera década posterior al acuerdo (Devarajan y Mottaghi, 2015). Si se consideran dichas estimaciones, se puede pensar que la élite del poder iraní, que controla los sectores de la energía, la tecnología y los servicios, sería la más beneficiada de las condiciones económicas del pacto nuclear al tratarse del principal administrador de las proyecciones económicas globales, en particular de la interacción con empresas europeas en rubros energéticos, farmacéuticos y del sector alimentario.

En septiembre de 2015, empresas como Total, Royal Dutch Shell y Eni, entre otras, enviaron delegaciones para dialogar con la National Iranian Oil Company y discutir las leyes iraníes que prohíben la propiedad extranjera de recursos estratégicos. El interés fundamental de las empresas ya no radica en los barriles que se producen en el presente, sino en la concesión de reservas que se administren a futuro. Otra evidencia, en el sector farmacéutico, reside en el interés del grupo danés Novo Nordisk, principal proveedor mundial de insulina, en 
construir en Irán una planta con un costo de alrededor de 70 millones de euros a finales de 2015 (Ward, 2015). Además, una de las transacciones comerciales más grandes, en el marco del PAIC, fue el acuerdo para comprar 173 aeronaves a Airbus, 140 aviones a Boeing y otros 40 aviones a la europea ATR por un monto aproximado de 32000 millones de dólares, en el que la compañía Iran Air fue la más interesada para transformar y modernizar su flota (Dudley, 2017).

\section{Nuevas tensiones alrededor del PAIC ante la llegada de Donald Trump y la reactivación del eje Washington- Riad-Tel Aviv}

El PAIC estableció un periodo de 15 años para estabilizar las tensiones nucleares y convertir un conflicto estratégico en acuerdos económicos, técnicos y financieros con beneficios para Irán y el G5+1. El documento que entró en vigor en enero de 2016 fue resultado directo de más de diez años de esfuerzos diplomáticos en un contexto marcado por la "guerra contra el terrorismo" por un lado, y, por el otro, un respiro geopolítico en la zona ante un escenario regional desolador debido a la intensificación del conflicto en Siria (y luego el de Yemen). El anexo 5 del paIc estableció los parámetros necesarios para darle seguimiento al levantamiento de las sanciones y al congelamiento de las actividades nucleares iraníes en un proceso vigilado por el OIEA, que implementó nuevas formas de supervisión, como los sellados inteligentes de fibra óptica (que pueden enviar alertas si son manipulados), cámaras inteligentes de video, visitas recurrentes de altos funcionarios e inspectores del organismo, entre otros elementos viables y verificables (U.S. Departament of State, 2015b).

Una realidad es que el PAIC sentó las bases para controlar cualquier intento de desviar el programa nuclear iraní hacia fines militares, al tiempo que Teherán cedió buena parte de su capacidad disuasiva a cambio de transacciones económicas útiles 
para reactivar su economía. Sin embargo, también es cierto que Irán conservó sus instalaciones científicas, tecnológicas y militares intactas, así como el control del ciclo de combustible nuclear, lo que significó la preservación de toda la infraestructura estratégica que los líderes de la República Islámica podrían reactivar en caso necesario.

La llegada de Trump a la presidencia de Estados Unidos en enero de 2017 supuso un desafío directo al pacto y a la red de negocios que posibilitó. Todo ocurrió en un contexto de retórica racista contra las comunidades latina y musulmana en Estados Unidos, y particularmente con un matiz de iranofobia en la esfera pública global a través de múltiples actos en los que el nuevo mandatario se refirió a Irán como "un país en la mira” y como "una amenaza directa a la seguridad internacional” (Iran in crosshairs of new administration, 2017). Trump dijo, además, que el PAIC era "uno de los peores acuerdos suscritos por Estados Unidos en su historia” (Kwong, 2017) y, a los pocos meses de tomar el cargo, relacionó a Irán directamente con el Estado Islámico, como si se tratara del mismo fenómeno.

El primer viaje de Donald Trump en calidad de presidente de Estados Unidos tuvo como destinos Riad y Tel Aviv, lugares donde creó el denominado Global Center for Combating Extremist Ideology (Etidal Center), con una clara tendencia antiiraní, como dejó sentado en el discurso inaugural, al cual se unió el mandatario egipcio Abdel Fatah al Sisi (Othaimin, 2017). ${ }^{7}$ No está de más señalar que durante la $72^{\text {a }}$ Asamblea

${ }^{7}$ Un estudio de 2017 de la Fundación Watani al-Emarat, en cooperación con la Autoridad Reguladora de Telecomunicaciones, el Servicio al-Ameen y el Centro de Seguridad Electrónica de Dubai (DESC), mostró que el Estado Islámico había usado las redes sociales para reclutar a la mayoría de sus adeptos. También reveló que en Facebook y Twitter, tenía más de 90000 cuentas en árabe y 40000 en otros idiomas. Por lo tanto, para combatir el extremismo, el Centro Etidal tendría tres frentes: el intelectual, el multimedia y el digital. La esencia del Centro proviene de contar con numerosos expertos y especialistas internacionales destacados en el campo de la lucha contra el discurso de los medios extremistas, al tiempo que posee tecnología de alto nivel para combatir actividades extremistas a través de los medios convencionales y el espacio digital, lo que lo convierte en un espacio de espionaje sumamente fuerte 
General de Naciones Unidas, Trump volvió a acusar a Irán de ser una "dictadura corrupta y oculta detrás de una falsa democracia”, y dijo que había "convertido al país en un 'Estado canalla' con una economía destruida 'cuyas principales exportaciones eran la violencia, el derramamiento de sangre y el caos" (Mesa, 2017a).

Es de notar que estas declaraciones tuvieron la intención de escalar el conflicto de la agenda nuclear en un contexto regional donde ya reinaba la incertidumbre frente a nuevos ciclos de violencia en Yemen, la intensificación del combate contra el Estado Islámico en Iraq y Siria (a raíz de sus derrotas en Mosul y la debilidad en Raqqa y Deir ez-Zor), el aumento de tensiones entre Israel y Hezbollah por su diferendo territorial y la competencia regional entre Irán y Arabia Saudí en la región. Para estos momentos, también era sabido que Trump trataba de cumplir las promesas de campaña hechas a su base social en Estados Unidos, nombrando a David M. Friedman como embajador de Washington en Israel, actor que había solapado el cambio de la embajada estadounidense de Tel Aviv a Jerusalén en una declaración que se hizo pública el 6 de diciembre de 2017 y que causó un nuevo brote de violencia en la zona y alimentó la tendencia iranofóbica de la nueva administración estadounidense (Martínez, 2017).

Otra señal orientada a castigar a Irán para cosechar apoyo interno fue la incorporación de John Bolton como asesor de Seguridad Nacional de la Casa Blanca el 22 de marzo de 2018 (Jaffe y Dawsey, 2018). Es sabido que Bolton sirvió en la administración de George W. Bush en un importante puesto de control de armas y que tiene una postura antiiraní no sólo por sus declaraciones a favor de un cambio de régimen en Teherán, sino también por sus simpatías con la organización Moyahedin-e Jalq, un grupo opositor al gobierno iraní que tiene su sede en Washington y París y que ha sido catalogado

y disuasivo para cualquier actor que pretenda usar el ciberespacio en la zona del Golfo. 
por Teherán como grupo terrorista por diversos asesinatos en contra de prominentes figuras políticas del establishment iraní desde la década de 1980.

Con lo anterior, el tema nuclear aceleró las conversaciones políticas y militares entre Tel Aviv y Riad en aras de alcanzar un contrapeso a la influencia que Irán había conseguido en Iraq, Líbano, Siria y Yemen. Además, el Departamento de Defensa de Estados Unidos y Arabia Saudí habían firmado una promesa de inversión en un paquete de 380000 millones de dólares, por lo que era lógico pensar que los movimientos y la retórica de Trump contra el pacto nuclear tenderían a materializar dicha inversión.

No obstante, como en múltiples ocasiones, Trump contradijo a los miembros de su gabinete, como a James Mattis, quien había afirmado públicamente que era "del interés estadounidense seguir con lo acordado por el pacto nuclear" (GibbonsNeff y Sanger, 2017). La administración tomó, sin embargo, la decisión de abandonar el acuerdo el 8 de mayo de 2018. Mientras $67 \%$ de los seguidores de Trump consideraban negativos instrumentos como los acuerdos de libre comercio, el PAIC aún gozaba de buena reputación no sólo entre la sociedad estadounidense, sino también globalmente, según una encuesta de finales de 2017 (McCarthy, 2017). Ejemplo de lo anterior es que el PAIC contó con el visto bueno del csNu y del Parlamento Europeo, recibió la certificación del Organismo Internacional de Energía Atómica en 30 ocasiones y tuvo el beneplácito de la comunidad científica que pugnaba por la no proliferación nuclear en el mundo, al grado de haber sido nominado al Premio Nobel de la Paz en 2017 (Garduño, 2017).

Lo que Trump argumentó para salir del PAIC fue el programa de misiles de Irán, que desde un principio quedó fuera de las negociaciones por considerarse parte de la soberanía iraní, y que Teherán no sometería a inspecciones por cuestiones de seguridad nacional. Como se ha señalado, el Consejo de Seguridad de Naciones Unidas votó la resolución 2231, que, en su párrafo 3 del anexo B, estableció que "Irán quedaría obligado 
a no emprender ninguna actividad relacionada con misiles balísticos capaces de transportar armas nucleares", cuestión que Trump utilizó primero para pugnar por una "descertificación" en octubre de 2017, cuando Teherán realizó una prueba exitosa del nuevo misil bautizado como "Jorramshahr", y luego para anunciar su salida definitiva del acuerdo, siete meses después (Garduño, 2017). ${ }^{8}$

Con la salida del pacto nuclear, Estados Unidos puso en marcha la denominada "estrategia de máxima presión" contra Irán en Medio Oriente, que nada tiene que ver con el enriquecimiento de uranio y que no cuenta con un objetivo claro, pues las limitaciones de la intervención militar, del cambio de régimen e incluso de la efectividad de las sanciones siguen presentes al tiempo de escribir estas líneas. De hecho, la explicación más lógica de esta decisión podría encontrarse en la búsqueda de fortalecimiento interno de Trump, pensando en su reelección, por una parte, y, por la otra, en debilitar la economía iraní para frenar el apoyo a grupos aliados en las crisis más agudas que vive el Medio Oriente, particularmente en Yemen, donde enfrenta la influencia saudí, y en Siria, por la presencia israelí. ${ }^{9} \mathrm{La}$ evidencia para este argumento radica en el anuncio del secretario de Estado Mike Pompeo del 21 de mayo de 2018, cuando amenazó a Irán con imponer "las sanciones más grandes en la

${ }^{8}$ La prueba de este misil debe contextualizarse en medio de la retórica antiiraní, aunada a nuevas acciones contra los Pasdaran, a quienes Washington catalogó como "patrocinadores del terrorismo" al relacionarlos en más de una ocasión con exportaciones de equipo prohibido a grupos armados en Yemen (Reuters, 2017). Hay que mencionar que el conflicto en Yemen fue intensificándose desde la intervención saudí en 2015, y que los grupos apoyados por Irán comenzaron a hacer pruebas exitosas de lanzamientos de cohetería y misiles de corto y mediano alcance a territorio saudí, como la del 4 de noviembre de 2017, que alcanzó el aeropuerto internacional Rey Jaled en Riad y dejó una fuerte duda sobre las capacidades reales y la efectividad de los sistemas de defensa aérea del reino saudí (Mesa, 2017b).

${ }^{9}$ Explotar el factor externo iraní es útil para mantener el apoyo de grandes empresarios antiiraníes que ayudaron a financiar su campaña, como Sheldon Adelson, Bernard Marcus y Paul Singery, quienes, junto con el lobby sionista cristiano que constituye buena parte de su base social, fungirían como sus mayores fortalezas en caso del destape de críticas más profundas a su gobierno en lo que va de su administración. 
historia" mediante la exposición de 12 demandas básicas para negociar un "verdadero acuerdo nuclear" (Pompeo threatens Iran with strongest sanctions in history, speeding U.S. march toward war, 2018). Ciertamente, entre las exigencias de Pompeo se mencionó el acceso irrestricto a los sitios nucleares de Irán y el cierre de su reactor de agua pesada, pero se enfatizó en factores extranucleares, como el fin del apoyo armado y económico a los hutíes en Yemen, el cierre de su programa de misiles, su salida de Siria y Afganistán, el cese de actividades de las fuerzas Al-Quds e, incluso, el alto a supuestas amenazas a países aliados de Estados Unidos en la región, como Israel y Arabia Saudí (U.S. Department of State, 2018).

A pesar de que esta estrategia de corto plazo ha ido acompañada de una nueva ola de iranofobia — que va desde la ruptura de relaciones diplomáticas de Marruecos con Irán por una supuesta colaboración entre Hezbollah y el Frente Polisario, la decisión de un juez en Nueva York que culpa a Irán por su presunta responsabilidad en los sucesos del 9/11, hasta los documentos que presentó Netanyahu como evidencia de las supuestas mentiras de Irán respecto a su programa nuclear (Iran Nuclear Programme Status, 2012)_, la Unión Europea, Rusia y China anunciaron su disposición a permanecer en el acuerdo pese a la salida de Estados Unidos. Entre las razones que explican por qué Europa se alineó con Rusia y China en esta cuestión, se tienen que ponderar los componentes económicos y la necesidad de mantener los contratos de las compañías involucradas, pese a las amenazas de Trump de reimponer sanciones y restricciones al mercado norteamericano a toda compañía que haga tratos con Irán. La estrategia de Washington, Riad y Tel Aviv generará sin duda un nuevo debate en la esfera pública iraní, debate que ya comenzó en las sesiones del parlamento y que coadyuva a fortalecer a los sectores más conservadores del faccionalismo iraní, a reserva del futuro del pacto nuclear, como se le ha conocido desde julio de 2015. 


\section{Conclusiones}

El PAIC ha sido una solución política y financiera que responde a un problema estratégico y geopolítico más que a una cuestión relacionada estrictamente con la energía nuclear en Irán, elemento que es parte de la ecuación, pero que no determina la conducta de actores regionales que se han opuesto al acuerdo, particularmente Arabia Saudí e Israel, que han visto en el gobierno de Trump un nuevo impulso a sus intereses regionales en Medio Oriente en general y en el golfo Pérsico en particular.

El camino para alcanzar el convenio no fue fácil. Las negociaciones incluyeron minuciosas cuestiones técnicas y de seguridad que prácticamente congelaron el programa nuclear iraní sin desmantelar su capacidad estratégica y disuasiva, lo que se convirtió en un instrumento multilateral de ganarganar, como lo evidenciaron los acuerdos y los negocios en los sectores automotriz, aéreo, petrolero y farmacéutico entre todos los actores (incluido Estados Unidos). Estos avances, al escribir estas líneas, se pasan por alto en la nueva administración estadounidense y han dejado que las garantías del pacto se desvanezcan en medio de una nueva retórica de amenazas, islamofobia e iranofobia que intentan allanar el camino para desmantelar el PAIC antes de que termine su vigencia de 15 años, tal como lo estipula el documento.

Aunque la dificultad de una intervención militar directa en Irán y la poca efectividad de las sanciones durante la primera década del siglo Xxi mostraron parte del camino para construir un entendimiento diplomático entre Irán y los países del G5 +1, la firma del PAIC no se entiende sin el clima de incertidumbre que las protestas populares árabes provocaron y que obligó a las partes en disputa a cambiar sus maniobras mediáticas y a flexibilizar su posición en la mesa de negociaciones para evitar que la cuestión nuclear complejizara aún más la crisis en Medio Oriente tras las movilizaciones sociales. No fue la disuasión de la intervención militar, sino su inoperabilidad, ni la efectividad de las sanciones, sino su insuficiencia, lo que cimentó 
el camino para el pacto nuclear con Irán en el contexto de la sacudida social que provocó la salida de millones de jóvenes en todo Medio Oriente y luego en Europa como un efecto claro de la crisis global de 2008. Por supuesto que los esfuerzos de Obama y Rohaní fueron fundamentales para instrumentar el acuerdo en sus respectivas opiniones públicas, pero es necesario ubicar dichos esfuerzos en las esferas regionales y globales para entender mejor el proceso de negociación y su éxito en julio de 2015.

Pese a lo anterior, la situación en la región no es mejor que cuando estallaron las protestas. Las administraciones de Israel y Arabia Saudí se han empoderado con la llegada de Trump al plantear el objetivo claro de lastimar el PAIC con la salida de Estados Unidos, la ampliación de las exigencias contra la República Islámica y la adición de temas que no fueron incluidos en 2015, como las inspecciones a instalaciones militares, y particularmente la revisión del programa de misiles de corto y mediano alcance, algo que para Teherán será muy difícil de aceptar. Al mismo tiempo, es evidente que Riad, Tel Aviv y Washington intentan minar la influencia de Irán en varios países árabes después de años de conflicto, en particular en algunas zonas de Iraq, Siria, Yemen, Líbano y Palestina, y que las amenazas en el contexto de "la cuestión nuclear" serán el nuevo guion de esta etapa del conflicto.

No está de más señalar que en las declaraciones de Trump respecto al PAIc hay un doble rasero, pues mientras Washington apela a la desnuclearización de Irán y de Corea del Norte, no condena la negación de Israel a firmar instrumentos multilaterales como el TNP y mucho menos a su desnuclearización. Estados Unidos critica el programa de misiles de Irán, pero no señala el riesgo que implican los programas de misiles de países como India o Pakistán, y aunque usa la prueba balística iraní como justificación para romper con el PAIC por considerarlo "uno de los peores tratados firmados por Estados Unidos", Trump no dice nada de la violencia ejercida en Yemen o Gaza a manos de sus aliados regionales. 
Una clave importante para el futuro inmediato del PAIC será estudiar la capacidad de Estados Unidos para convencer al resto de las partes, principalmente Francia y Alemania, de abandonar el pacto con Irán de manera definitiva, o al menos para que busquen un acuerdo suplementario que involucre el tema del programa de misiles a sabiendas de que Teherán se negará automáticamente, haciendo que esta nueva estrategia de presión provoque el desgaste de Irán por medio de una fuerte presión no sólo en la esfera pública trasnacional, sino también en los teatros de operaciones donde se llevan a cabo los enfrentamientos con grupos proxys en escenarios sensibles como Siria, Iraq, Yemen y potencialmente Líbano.

\section{Referencias}

Abrahamian, E. (2015). The coup: 1953, the CiA, and the roots of modern U.S.-Iranian relations. Nueva York, NY: The New Press.

Alandete, D. (16 de julio de 2012). Un barco militar de EEUU abre fuego contra un bote en aguas del golfo Pérsico. El País. Recuperado de https://elpais.com/internacional/2012/07/16/ actualidad/1342458571_053421.html

Alizadeh, P. (2014). The political economy of petro populism and reform, 1997-2011. En P. Alizadeh y H. Hakimian (Eds.), Iran and the global economy: Petro populism, Islam and economic sanctions (pp. 76-111). Abingdon: Routledge.

Associated Press (18 de julio de 2012). Israel blames Iran for deadly attack on bus of Israeli youth visiting Bulgaria. New York Daily Neres. Recuperado de http://www.nydailynews.com/ news/world/explosion-targets-bus-young-israeli-tourists-bulgaria-article-1.1116853

BARZEGAR, K. (2008a). Iran's foreign policy in post-invasion Iraq. Middle East Policy, 15(4), 47-58. https://doi.org/10.1111/j.14754967.2008.00368.x

BARzegar, K. (8 de diciembre de 2008b). Mu'amala Bozorg: Iran va Obama [Obama e Irán: el gran acuerdo], Tabnak. Recuperado de

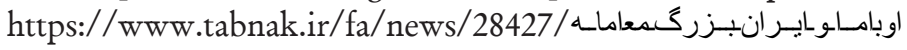


Barzegar, K. (2010). Balance of power in the Persian Gulf: An Iranian view. Middle East Policy, 17(3), 74-87. https://doi. org/10.1111/j.1475-4967.2010.00452.x

BENARI, E. (6 de noviembre de 2012). Netanyahu: I will attack Iran alone if necessary. Israel National Neres. Recuperado de http:// www.israelnationalnews.com/News/News.aspx/161749

Bergman, R. (10 de junio de 2009). Letter from Tel Aviv: Netanyahu's Iranian dilemma. The limits of the military option against Iran. Foreign Affairs. Recuperado de https://www.foreignaffairs. com/articles/middle-east/2009-06-10/letter-tel-aviv-netanyahus-iranian-dilemma

Borger, J. y Dehghan, S. K. (10 de noviembre de 2013). Geneva talks end without deal on Iran's nuclear programme. The Guardian. Recuperado de https://www.theguardian.com/ world/2013/nov/10/iran-nuclear-deal-stalls-reactor-plutoniumfrance

Bush, G. W. (2010). Decision points. Nueva York, NY: Crown Publishers.

Cordesman, A. (2007). Iran, oil and Strait of Hormuz. Washington: Center for Strategic and International Studies. Recuperado de https://www.csis.org/analysis/iran-oil-and-strait-hormuz

Charbonneau, L. (24 de septiembre de 2012). In New York, defiant Ahmadinejad says Israel will be "eliminated". Reuters. Recuperado de https://www.reuters.com/article/us-un-assemblyahmadinejad/in-new-york-defiant-ahmadinejad-says-israel-willbe-eliminated-idUSBRE88NOHF20120924

CHIN, S. y Lincy, V. (14 de julio de 2015). How will inspections work in Iran under the nuclear deal? Iran Watch. Recuperado de http:// www.iranwatch.org/our-publications/nuclear-iran-weekly/ how-will-inspections-work-iran-under-nuclear-deal

Chumley, C. K. (6 de febrero de 2013). Shoe thrown at Mahmoud Ahmadinejad, similar to incident with George W. Bush, during visit to Egypt. The Washington Times. Recuperado de http:// www.washingtontimes.com/news/2013/feb/6/iran-presidentseeks-alliance-egypt/

Dabashi, H. (2011). The Green Movement in Iran. Nuevo Brunswick, NJ: Transaction Publishers.

DAVENPORT, K. (3 de diciembre de 2015). Iran dismantling centrifuges, IAEA says. Arms Control Association. Recuperado de https:// 
www.armscontrol.org/ACT/2015_12/News/Iran-DismantlingCentrifuges-IAEA-Says

Dehghan, S. K. (17 de octubre de 2012). Iran sanctions 'putting millions of lives at risk'. The Guardian. Recuperado de https:// www.theguardian.com/world/2012/oct/17/iran-sanctionslives-at-risk

Devarajan, S. y Mottaghi, L. (2015). Middle East and North Africa Quarterly Economic Brief, July 2015: Economic implications of lifting sanctions on Iran. Washington, DC: World Bank. https:// doi.org/10.1596/978-1-4648-0702-2

Dudley, D. (28 de junio de 2017). How Iran is transforming its aviation industry with multi-billion dollar orders for hundreds of jets. Forbes. Recuperado de https://www.forbes.com/sites/ dominicdudley/2017/06/28/iran-transforming-aviationindustry/\#4fc081f03610

ERAmi, N. y Keshavarzian, A. (2015). When ties don't bind: Smuggling effects, bazaars and regulatory regimes in postrevolutionary Iran. Economy and Society, 44(1), 110-139. https://doi.org/10.1080/ 03085147.2014.909986

Espinosa, Á. (11 de enero de 2012). Un científico iraní muere en ataque bomba en Teherán. El País. Recuperado de http:// internacional.elpais.com/internacional/2012/01/11/actuali$\mathrm{dad} / 1326271250$ 879920.html

ForozAn, H. (2016). The military in post-revolutionary Iran: The evolution and roles of the revolutionary guards. Londres: Routledge. https://doi.org/10.4324/9781315691640

Full text Obama gives a speech about the nuclear deal (5 de agosto de 2015). The Washington Post. Recuperado de https:// www.washingtonpost.com/news/post-politics/wp/2015/08/ 05/text-obama-gives-a-speech-about-the-iran-nuclear-deal/?utm term $=.68 \mathrm{e} 704 \mathrm{a} 82 \mathrm{eef}$

GARDUÑO, M. (2011). La crisis de confianza y el balance de poder entre Irán y las grandes potencias en la cuestión nuclear. Revista de Estudios Internacionales Mediterráneos, (10), 72-81. Recuperado de https://revistas.uam.es/index.php/reim/article/view/850/838

GARDUÑO, M. (2012). Le modèle des pâsdârân-artesh: préoccupations internes et défis externes dans le cadre du printemps arabe. Maghreb-Machrek, 214(4), 71-84. https://doi.org/10.3917/machr.214.0071 
GarduÑo, M. (6 de noviembre de 2017). ¿Puede el pacto nuclear con Irán sobrevivir a Donald Trump? Foreign Affairs Latinoamérica. Recuperado de http://revistafal.com/puede-el-pacto-nuclearcon-iran-sobrevivir-a-donald-trump/

GibBons-NefF, T. y Sanger, D. E. (3 de octubre de 2017). Mattis contradicts Trump on Iran deal ahead of crucial deadline. The New York Times. Recuperado de https://www.nytimes.com/2017/ 10/03/world/middleeast/mattis-iran-deal-trump.html

GuPTA, S. (23 de julio de 2015). Oman: The unsung hero of the Iranian nuclear deal. Foreign Policy Journal. Recuperado de https://www. foreignpolicyjournal.com/2015/07/23/oman-the-unsung-heroof-the-iranian-nuclear-deal/

Habibi, N. (2013). The economic legacy of Mahmoud Abmadinejad (Middle East Brief, 74). Waltham, MA: Brandeis University. Recuperado de http://www.brandeis.edu/crown/publications/ meb/MEB74.pdf

Hakimian, H. (2014). Iran's free trade zones. Back doors to the international economy? En P. Alizadeh y H. Hakimian (Eds.), Iran and the global economy: Petro-populism, Islam and economic sanctions (pp. 102-123). Abingdon: Routledge.

Handmade carpet exporters look to China market (6 de enero de 2011). Iran Daily. Recuperado de http://old.iran-daily.com/ 1389/10/16/MainPaper/3864/Page/4/MainPaper_3864_4.pdf

Iran to sue European refusal to refuel planes: Airline (31 de octubre de 2010). Al Arabiya. Recuperado de https://www.alarabiya. net/articles/2010/10/30/124253.html

Iranian airplane crashes expose weaknesses in 'self-sufficiency' (21 de agosto de 2014). Al Monitor. Recuperado de http://www. al-monitor.com/pulse/originals/2014/08/iran-airplane-crashsanctions-parts-sufficiency.html

Iran in crosshairs of new administration (22 de enero de 2017). The Real News Network. Recuperado de https://therealnews.com/ stories/rerlich0120trump

Irán libera a los infantes de la Marina de EE.UU. detenidos en el Golfo Pérsico (13 de enero de 2016). BBC. Recuperado de https://www. bbc.com/mundo/noticias/2016/01/160111_iran_eeuu_soldados_captura_jg

Iran Nuclear Programme Status [cable de información] (22 de octubre de 2012). Recuperado de https://www.aljazeera.com/ 
news/2015/02/leaks-netanyahu-misled-iran-nuclear-programmeguardian-iran-nuclear-speech-2012-150218165622065.html

Jaffe, G. y Dawsey, J. (22 de marzo de 2018). Trump names former ambassador John Bolton as his new national security adviser. The Washington Post. Recuperado de https://www. washingtonpost.com/world/national-security/trump-namesformer-ambassador-john-bolton-as-his-new-national-securityadviser/2018/03/22/aa1d19e6-2e20-11e8-8ad6-fbc50284fce8_story. html? noredirect $=$ on\&utm term $=. \mathrm{dd} 3 \mathrm{c} 8592 \mathrm{ee} 08$

Joyce, M. (2012). Babrain from the twentieth century to the Arab Spring. Nueva York, NY: Palgrave Macmillan. https://doi. org/10.1057/9781137031792

KaHL, C. H. (2012). Not time to attack Iran: Why war should be a last resort. Foreing Affairs, 91(2), 166-173. Recuperado de https://www.foreignaffairs.com/articles/iran/2012-01-17/nottime-attack-iran

KATZMAN, K. (2 de diciembre de 2011). Iran sanctions (Congressional Research Service Report for Congress, RS20871). Recuperado de https://www.hsdl.org/?view\&did=695451

KhameneI, A. (14 de marzo de 2014). Yalesteh tabiin siyaset-ha ye Eqtesad- e Muqawamati. [Reunión explicativa de las políticas de la economía de la resistencia]. Khamenei.ir. Recuperado de http://farsi.khamenei.ir/newscontent?id $=25775$

Kroenig, M. (2012). Time to attack Iran. Foreign Affairs, 91(1), 7686. Recuperado de https://www.foreignaffairs.com/articles/ middle-east/2012-01-01/time-attack-iran

Kwong, M. (21 de septiembre de 2017). Trump calls the Iran nuclear deal 'the worst' but exiting it could be worse still. $C B C$ Neres. Recuperado de http://www.cbc.ca/news/world/trump-rouhaniiran-nuclear-deal-un-1.4301571

Martínez, J. (6 de diciembre 2017). Trump siembra la discordia al reconocer Jerusalén como capital de Israel. El País. Recuperado de https://elpais.com/internacional/2017/12/06/estados_unidos/1512579049_062317.html

McCarthy, N. (12 de octubre de 2017). Poll: Most Americans support the 2015 Iran nuclear deal. Forbes. Recuperado de https://www.forbes.com/sites/niallmccarthy/2017/10/12/ poll-most-americans-support-the-2015-iran-nuclear-deal-infographic/\#456bb0207d93 
Mesa, L. (2009). Las políticas de Bush y Obama hacia la República Islámica de Irán. La centralidad del factor nuclear. Foro Internacional, 49(4), 832-863. Recuperado de https://forointernacional. colmex.mx/index.php/fi/article/view/1982/1972

Mesa, L. (30 de septiembre de 2017a). Donald Trump ataca de Nuevo al acuerdo nuclear con Irán. Siempre. Recuperado de http:// www.siempre.mx/2017/09/donald-trump-ataca-de-nuevo-alacuerdo-nuclear-con-iran/

Mesa, L. (11 de noviembre de 2017b). Irán y Arabia Saudita: incrementan su antagonismo estratégico. Siempre. Recuperado de http://www.siempre.mx/2017/11/iran-y-arabia-sauditaincrementan-su-antagonismo-estrategico/

MotAamen-SAmadian, S. (2014). The role of government in the Iranian banking system, 2001-11. En P. Alizadeh y H. Hakimian (Eds.), Iran and the global economy: Petro-populism, Islam and economic sanctions (pp. 136-138). Abingdon: Routledge.

Othaimin, I. (7 de julio de 2017). Various dimensions of the fight against extremist ideology. Recuperado de http://www.riyadhvision.com.sa/2017/07/07/various-dimensions-of-the-fightagainst-extremist-ideology/

PARSI, T. (2007). Treacherous alliance: The secret dealings of Israel, Iran, and the United States. New Haven, CT: Yale University Press.

Pompeo threatens Iran with strongest sanctions in history, speeding U.S. march toward war (22 de mayo de 2018). Democracy Now. Recuperado de https://www.democracynow.org/2018/5/22/ pompeo_threatens_iran_lays_out_unattainable

RAHIMI, B. (2012). Iran's declining influence in Iraq. The Washington Quarterly, 35(1), 25-40. https://doi.org/10.1080/016366 OX.2012.641917

Reuters (26 de octubre de 2010). Iran exports gasoline to Iraq, Afghanistan, Armenia. Reuters. Recuperado de http://www.reuters. com/article/us-iran-gasoline-export-idUSTRE69P31T20101026

Reuters (20 de noviembre de 2017). U.S. sanctions individuals, entities for Iran-linked counterfeiting. Reuters. Recuperado de https://www.reuters.com/article/us-usa-iran-sanctions/us-sanctions-individuals-entities-for-iran-linked-counterfeitingidUSKBN1DK28Q? il = 0

Rouhani: Iran has achieved all its 4 objectives in nuclear talks (15 de julio de 2015). Teheran Times. Recuperado de http://www. 
tehrantimes.com/news/248035/Rouhani-Iran-has-achieved-allits-4-objectives-in-nuclear-talks

SALEHI-IsfaHANI, D. (23 de enero de 2013). Is Iranian hyperinflation a mirage? Al Monitor. Recuperado de http://www.al-monitor. com/pulse/originals/2013/01/hyperinflation-iran-manti-teo. html

SAmore, G. (Ed.) (2015). Sanctions against Iran: A guide to targets, terms, and timetables. Addendum to: Decoding the Iranian nuclear deal (April 2015). Cambridge, MA: Belfer Center for Science and International Affairs. Recuperado de http://belfercenter.ksg. harvard.edu/files/Iran\%20Sanctions.pdf

SANDLER, T. y George, J. (2016). Military expenditure trends for 1960-2014 and what they reveal. Global Policy, 7(2), 174-184. https://doi.org/10.1111/1758-5899.12328

TAROCK, A. (2016). The nuclear deal: Winning a little, losing a lot. Third World Quarterly, 37(8), 1408-1424. https://doi.org/10.10 80/01436597.2016.1166049

TAYLOR, N. (12 de enero de 2013). The Middle East needs to be a nuclear weapons free zone. Al Jazeera. Recuperado de http://www. aljazeera.com/indepth/opinion/2013/01/2013141248927573. html

Torabian, S. y Abalakina, M. (2012). Attitudes toward war in the United States and Iran. Iranian Studies, 45(4), 463-478. https:// doi.org/10.1080/00210862.2012.673825

Toussaint, E. (12 de enero de 2012). Part II: The global crisis that preceded the Arab Spring, the Indignados, and the Occupy Wall Street movement. International Viewpoint, (444). Recuperado de http://internationalviewpoint.org/spip.php?article2442

Una guerra entre Irán y Estados Unidos en el Golfo Pérsico [Yang Iran va Amrika dar Khalij Farsi] (2 de noviembre de 1987). Fatehan.ir. Recuperado de http://www.fatehan.ir/page.aspx?pid =331

UNCTAD (United Nations Conference on Trade and Development) (2013). Iran, Islamic Republic of. En International Investment Agreements Navigator [base de datos]. Recuperado de https:// investmentpolicy.unctad.org/international-investment-agreements/countries/98/iran-islamic-republic-of

United Nations Office for Disarmament Affairs (5 de marzo 1970). Treaty of non-proliferation of nuclear weapons. Recuperado de http://disarmament.un.org/treaties/t/npt 
U.S. Department of State (3 de agosto de 2007). U.S. and India released text of 123 agreement. Recuperado de https://2001-2009.state. gov/r/pa/prs/ps/2007/aug/90050.htm

U.S. Department of State (13 de enero 2015a). The United States and Pakistan: Strong and enduring cooperation [comunicado de prensa]. Recuperado de https://2009-2017.state.gov/r/pa/prs/ ps/2015/01/235883.htm

U.S. Departament of State (14 de julio de 2015b). Joint comprehensive plan of action [comunicado de prensa]. Recuperado de https://2009-2017.state.gov/p/nea/rt/jcpoa/index.htm

U.S. Department of State (21 de mayo de 2018). After the deal. A new Iran strategy. Recuperado de https://www.state.gov/after-thedeal-a-new-iran-strategy/

VAEZ, A. y Sadjadpour, K. (2013). Iran's nuclear odyssey: Costs and risks. Washington, DC: Carnegie Endowment for International Peace.

Ward, A. (22 de septiembre de 2015). Novo Nordisk to build $€ 70 \mathrm{~m}$ plant in Iran. Financial Times. Recuperado de http://www. ft.com/intl/cms/s/0/444a81d4-6114-11e5-9846-de406ccb37f2. html

Wood, T. W., Milazzo, M. D., Reichmuth, B. A. y Bedell, J. (2007). The economics of energy independence for Iran. The Nonproliferation Review, 14(1), 89-112. https://doi.org/10.1080/ 10736700601178572

Moisés Garduño García es doctor en estudios árabes e islámicos contemporáneos por la Facultad de Filosofía de la Universidad Autónoma de Madrid y maestro en estudios de Asia y África por El Colegio de México. Realizó una estancia posdoctoral en el Centro de Investigaciones y Estudios Superiores en Antropología Social, sede Occidente, y actualmente es profesor de tiempo completo en la Facultad de Ciencias Políticas y Sociales de la Universidad Nacional Autónoma de México, donde imparte clase en los programas de licenciatura y posgrado en relaciones internacionales. Es miembro del Sistema Nacional de Investigadores del Consejo Nacional de Ciencia y Tecnología, México, en el nivel 1, y en 2018 obtuvo el reconocimiento Dis- 
tinción Universidad Nacional para Jóvenes Académicos, en el área de docencia en Ciencias Sociales, que otorga la Universidad Nacional Autónoma de México.

https://orcid.org/0000-0002-3407-6578 mgarduno@politicas.unam.mx 
